\title{
PIV measurement of buffer and logarithmic layers with detached eddies which mimics the neutral atmospheric surface layer
}

\author{
Y. Hattori ${ }^{1 *}$, Hitoshi Suto ${ }^{1}$, Keisuke Nakao ${ }^{1}$, Hiromaru Hirakuchi ${ }^{1}$ \\ ${ }^{1}$ Central Research Institute of Electric Power Industry, Civil Eng Lab, Abiko, Japan \\ yhattori@criepi.denken.or.jp
}

\section{Motivation}

Accurate comprehension of turbulence characteristics in the atmospheric surface layer (ASL) under near neutral conditions, which is a lower part of the atmospheric boundary layer and a very high-Re number flow, is critically required in view of the increasing and broadening use of numerical weather prediction models. The models need to estimate turbulence fluxes of momentum, heat and moisture in the ASL as boundary conditions. On the other hand, observations (Högström 1990, Drobinski et al. 2007) have revealed that the fluxes under near-neutral conditions are often inconsistent with Monin-Obukhof theory, which has been widely used in models. The observations were conducted over flat surfaces with homogeneous roughness, and thus the violation from the theory might not be due to the underlying surface conditions. Thus, aiming to investigate an origin of the violation from the theory, we have carried out a wind tunnel experiment on the logarithmic layer along a smooth flat wall with a larger-scale disturbance, which mimics the near-neutral atmospheric surface layer (Hattori et al. 2010). In the present study, we especially examine a PIV measurement with a long-distance microscope lens to discuss the interaction of turbulences structures between buffer and logarithmic layers, which must give a clue on Reynolds number effects

\section{Experimental apparatus and procedure}

We used the same experimental technique of our previous study (Hattori et al. 2010), except velocity measurements. The experiment was conducted in an open-circuit wind tunnel at the Central Research Institute of Electric Power Industry (CRIEPI). The test section is $1000 \times 1000 \mathrm{~mm}^{2}$ in area and $6200 \mathrm{~mm}$ long, and the walls of the test section are made of smooth flat wood walls. An active turbulence grid (Makita et al. 1987), installed at the front of the test section, was used to control the turbulence characteristics in the logarithmic layer; the active turbulence grid composed of rotating grid bars with attached triangular agitator wings, stepping motors located at the end of each grid bards outside the wind tunnel and a controller. The velocity at the centerline of the test section was set to $5 \mathrm{~m} \cdot \mathrm{s}^{-1}$ to obtain the fully-developed turbulence boundary layer at the measuring location, which was fixed at the downstream distance from the active turbulence grid of $4180 \mathrm{~mm}$. The logarithmic layer height, $h_{s}$, and the friction velocity, $u_{\tau}$, which are characteristic length and velocity for the logarithmic layer flow, were $70 \mathrm{~mm}$ and $0.2 \mathrm{~m} \cdot \mathrm{s}^{-1}$, respectively.

In the present study, the velocity vectors were measured by using a PIV technique with a long-distance microscope lens. Olive-oil mists added to the wind field were illuminated by light sheet discharged from an YAG laser system, the output of which is $200 \mathrm{~mJ} /$ pulse. Particle-containing flow images in the streamwisevertical $(x-z)$ plane were captured by a CCD camera $(2 \mathrm{k} \times 2 \mathrm{k}$ pixels $)$ with a long-distance microscope lens to ensure spatial resolutions for capturing small turbulence eddies, which are generated near the wall. The distance between the lens and the object plane was set to $800 \mathrm{~mm}$, giving the physical size of measuring area was $10.8 \times 10.8 \mathrm{~mm}^{2}$. The time interval between the two pulsed illuminations was set at $20 \mu$ s to keep the maximum displacement of successive particle images below the half of the interrogation windows size. The velocity vectors were calculated using a cross-correlation method with interrogation windows of a size of $32 \times 32$ pixels. The overlap ratio was $50 \%$, and the 16384 velocity vectors were obtained in each pair of images, which gives the spatial resolutions normalized with inner parameters of $\Delta^{+}=1.12$. 


\section{Results}

Figure 1 depicts an example of visualized flow fields with calculated velocity vectors. The particles are appropriately added in flow fields, and the velocity vectors are obtained even in the vicinity of the surface of the wall. The velocities become very small near the surface with the non-slip conditions, and become large with organized motions above the near-wall region. Figure 2 shows the profile of time-averaged streamwise velocity normalized with inner parameters, with the lines for $U^{+}=z^{+},=(1 / 0.41) \ln z^{+}+5.0$. This profile shows the present PIV firmly captures the velocity fields of buffer and logarithmic layers. The profile agrees well with that for logarithmic layer for $z^{+}>30$ and approaches that for viscous sublayer for $z^{+}<30$.

More detailed experimental results on the structural characteristics will be presented in a presentation to understand fundamental characteristics of turbulence structure in the surface layer. Such results also provide a deep insight into coherence structures of a wall turbulence under high Reynolds number conditions; the ASL mimicked in the present study easily yields turbulence flows, the Reynolds number of which is much larger than those with wind tunnels. Indeed, a renowned observation campaign at the Surface Layer Turbulence and Environmental Science Test facility (Kunkel and Marusic 2006) used to examine the turbulence structures under very high Reynolds number conditions and have revealed the existence of very large-scale organized motions in the logarithmic layer (Hutchins and Marusic 2007, Marusic et al. 2010).

\section{References}

Drobinski, P, Carlotti P, Redelsperger J-L, Banta RM, Masson V and Newsom RK (2007) Numerical and experimental investigation of the neutral atmospheric surface layer. J Atmos Sci 64: 137-156

Hattori Y, Moeng C-H, Suto H, Tanaka N and Hirakuchi H (2010) Wind-tunnel experiment on logarithmiclayer turbulence under the influence of overlying detached eddies. Boundary-Layer Meteorol 134: 269-283

Högström U (1990) Analysis of turbulence structure in the surface layer with a modified similarity formulation for near neutral conditions. J Atmos Sci 47: 1949-1972

Hutchins N and Marusic I (2007) Evidence of very long meandering features in the logarithmic region of turbulent boundary layers. J Fluid Mech 579: 1-28

Makita H, Sassa K, Iwasaki T and Iida A (1987) Evaluation of the characteristic features of a large-scale turbulence field (1st report, performance of the turbulence generator). Trans JSME B 53: 3173-3179 (in Japanese)

Marusic I, mathis R and Huthins N (2010) Predictive model for wall-bounded turbulent flow. Science 329: 193-196

Kunkel GJ and Marusic I (2006) Study of the near-wall-turbulent region of the high-Reynolds-number boundary layer using an atmospheric flow. J Fluid Mech 548: 375-402

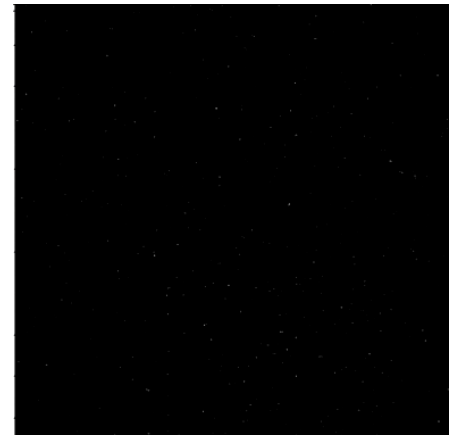

(a) Visualized image

(b) instantaneous velocity vectors

Figure 1: Example of image and instantaneous velocity vectors

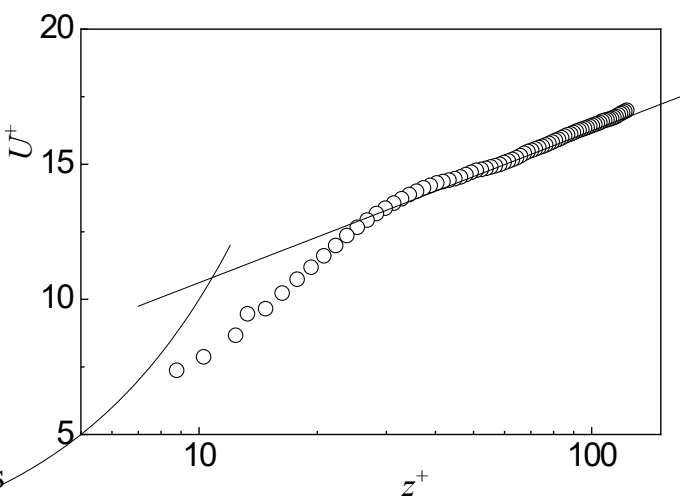

Figure2: Profile of time-averaged $U$ 